\title{
“OCUPARSE: Una propuesta de intervención con personas privadas de libertad basado en la ocupación"
}

\section{"OCUPARSE: An offer of intervention with imprisoned persons based on the occupation"}

\author{
Sandra Mella Díaz ${ }^{1}$, Vivian Villarroel Encina ${ }^{2}$, Rodrigo Sepúlveda ${ }^{3}$, Gonzalo \\ Guzmán Cáceres ${ }^{4}$
}

\section{Resumen:}

El artículo presenta un modelo de intervención con personas privadas de libertad desde la perspectiva ocupacional, desarrollado por la Escuela de Terapia Ocupacional de la Universidad de Chile en el marco de un convenio con Gendarmería de Chile. La propuesta metodológica contempló las fases de diseño e implementación, en la primera se indagó sobre el estado del arte en el tema, las actualización de fundamentos teóricos y consultas a expertos, el resultado de esta fase fue la elaboración de un módulo de intervención denominado "OCUPARSE" para ser aplicado por terapeutas ocupacionales, psicólogos o asistentes sociales de los equipos profesionales al interior de los centros privativos de libertad de Gendarmería de Chile. Posterior a la fase de diseño se realizó la implementación que incluyó una fase revisión por expertos, la edición del modulo, la aplicación de este a una muestra de personas privadas de libertad y posteriormente la capacitación de equipos profesionales multidisciplinarios.

Palabras claves: reinserción social, ocupación, personas privadas de libertad

\begin{abstract}
The article presents a model of intervention with imprisoned persons from the perspective occupational, developed by the School of Occupational Therapy at the University of Chile in the framework of an agreement with Chilean Gendarmerie. The proposed methodology included the design and implementation phases, the first inquired about the state of the art
\end{abstract}

\footnotetext{
${ }^{1}$ Terapeuta Ocupacional, Magíster en Integración de Personas con Discapacidad, Licenciada en Ciencia de la Ocupación, Profesor Asistente Universidad de Chile. Escuela de Terapia Ocupacional e-mail: smella@med.uchile.cl

${ }^{2}$ Terapeuta Ocupacional, Magíster en Educación Superior en Ciencias de la Salud $\mathbb{C}$, Licenciada en Ciencia de la Ocupación, Profesor Asistente Universidad de Chile. Escuela de Terapia Ocupacional e-mail: vvillarroel@med.uchile.cl

${ }^{3}$ Antropólogo, Profesor Asistente Universidad de Chile. Escuela de Terapia Ocupacional e-mail: rsepulveda@med.uchile.cl

${ }^{4}$ Psicólogo, Gendarmería de Chile, Departamento de Readaptación. Gobierno de Chile.

${ }^{5}$ Módulo de intervención solicitado por Gendarmería de Chile, 2007. Equipo Diseño: Sandra Mella Díaz, Vivian Villarroel Encina, Rodrigo Sepúlveda Prado, Equipo Revisor: Laura Rueda Castro y Alicia Valdés Rojas. Escuela Terapia Ocupacional. Facultad de Medicina. Universidad de Chile.
} 
on the theme, the updating of theoretical and consultation with experts, the outcome of this phase was the development of a module called the speech "OCUPARSE" to be implemented by occupational therapists, psychologists or social workers from professional equipment to the interior of the Gendarmerie de Chile Jails. After the design phase was the implementation phase, which included a review by experts, editing module, applying this to a sample of persons deprived of their liberty and then training for multidisciplinary teams of professionals.

Key words: social rehabilitation, occupation, imprisoned persons

\section{Introducción}

Muchas veces en las discusiones mediáticas se describe a los personas privadas de libertad, en adelante PPL, como objetos de sanción penal y por ello principalmente de control o bien como sujetos que deben recibir una intervención orientada a adquirir habilidades por medio de talleres que podrán ayudarles en su integración social al salir de los recintos penitenciarios.

Sin embargo, muchas de estas visiones y estrategias de intervención, carecen de una fundamentación coherente y rara vez son pensadas desde la vida cotidiana de las personas. El análisis desde la ocupación como un espacio de intervención, nos permite organizar el trabajo orientando las actividades y necesidades propias de estas personas, inmersos en un ambiente y una cultura particular que debe ser considerada en la intervención intra y extra penitenciaria.

La ocupación y la rutina ocupacional, se expresan habitualmente en todas las personas durante el desarrollo de las actividades cotidianas. El ser humano realiza un número importante de actividades en su vida, estas además tienen un patrón dependiendo de la edad y las circunstancias de la persona.

Según la American Occupational Therapy Association las actividades diarias desarrolladas por las personas se pueden clasificar en diversas áreas de Desempeño Ocupacional: Actividades de la Vida Diaria ( en adelante AVD) Básicas, AVD Instrumentales, Educación, Trabajo, Juego, Tiempo Libre y Participación Social (AOTA ,2002). Más allá de las actividades de la vida diaria, las ocupaciones son fenómenos complejos que están impregnadas en la vida de las personas y toman diversos visos dependiendo del momento de vida de esta persona y del contexto. (Gómez S, Rueda, L. \& Muñoz, C. 2002)

Toda persona tiene una conformación o estructura ocupacional determinada que está dada por las etapas del ciclo vital, motivaciones y exigencias del contexto, esta conformación no es rígida por el contrario constantemente esta cambiando, estos procesos adaptativos se dan en forma inconsciente la mayor parte del tiempo nos adaptamos sin pensarlo a las contingencias, existen sin embargo situaciones en que el cambio o estructura ocupacional requiere una organización más consciente y reflexiva en donde debemos valorar, priorizar y negociar las necesidades, exigencias e intereses. 
Westorp, P. , refiere que el equilibrio es clave entre y con los factores intrínsecos y extrínsecos o sea en relación a nuestras necesidades o intereses personales y de acuerdo a las demandas impuestas por la familia, la sociedad y la cultura. (Westorp, P. ,2003)

Las actividades de la vida diaria, tienen características muy diferentes en las personas privadas de libertad, la organización de estas esta relacionada directamente con los tiempos internos del recinto, en relación a esto cabe recordar que el significado de la ocupación se refiere a la experiencia subjetiva de la participación en las ocupaciones. Más aún las ocupaciones están simbólicamente constituidas en una cultura y son interpretadas en el contexto de la historia vital de los individuos. (Crepeau, 2003: 16).

El equilibrio ocupacional al interior de los recintos carcelarios no recibe la atención debida, el qué hacer y cómo hacerlo no es considerado, la rutina ocupacional no está organizada, cada persona y en la mayoría de los casos cada grupo establece las actividades, las normas y sus ritmos que muchas veces son partes de la misma cultura delictual, contribuyendo precisamente a lo que debemos evitar. En la cotidianeidad carcelaria se manifiesta la primacía de las acciones de control y manejo administrativo oscureciendo otro tipo de abordajes, que podrían tener un gran valor como estrategias intervención. En muchos casos también nos encontramos con la presencia de casos de una suerte de deprivación ocupacional que vulnera derechos inalienables del ser humano.

La ocupación constituye una fuente de bienestar y desarrollo cuando se puede vivir de manera plena. Como atributo propio del ser humano constituye un elemento esencial de la vida en sociedad y un derecho inalienable, es por ello que, aludiendo al concepto de justicia ocupacional el Módulo se propuso con un enfoque centrado en los principios y derechos de todo ser humano pensando en el desarrollo de oportunidades en las que las personas privadas precisamente de libertad, puedan involucrarse en acciones que se acerquen al ejercicio de éstos derechos. (Towsend, E. \& Wilcock, A. 2002)

Consciente de lo anterior la Escuela de Terapia de Terapia Ocupacional de la Universidad de Chile asumió el desafío de diseñar un modelo de intervención penitenciaria, por encargo de Gendarmería de Chile. La propuesta se fundamenta en los desarrollos de la Ciencia de la Ocupación y la Terapia Ocupacional. La intervención basada en la actividad con significado, apunta a favorecer cambios ocupacionales desde el espacio penitenciario, las relaciones familiares y favorecer la integración post egreso de las personas privadas de libertad.

La propuesta diseñada por el equipo a cargo finaliza en la creación de un módulo de intervención denominado Módulo de Integración Socio Ocupacional "OCUPARSE", y representa un acercamiento a las libertades fundamentales que cada individuo desarrolla en los contextos de la vida cotidiana y de participación pública. Por ello, se presenta como un proyecto que tiene la particularidad de eliminar barreras que obstaculizan los cursos de desarrollo vital en un ambiente de restricción, pues ofrece una alternativa de expresión y aprendizaje de actividades que fomentan el crecimiento personal y colectivo.

Por otro lado, la intervención a través de la ocupación permite garantizar el derecho a la integración post-penitenciaria, pues es a través de las ocupaciones que se produce la incorporación al orden social. La Ocupación constituye una nueva perspectiva de la 
integración social o rehabilitación, basada en la motivación intrínseca y el desarrollo ocupacional de sujeto a partir de sus intereses y su contexto cotidiano.

Gendarmería de Chile al incorporar esta propuesta en sus planes de trabajo apunta a aumentar la calidad de vida de personas privadas de libertad y favorecer la reintegración social una vez concluida su etapa de reclusión, el modelo y el manual desarrollado representan en este sentido un aporte significativo al desarrollo de esta línea de trabajo.

\section{METODOLOGÍA}

El proceso de elaboración del módulo implicó una coordinación constante entre el equipo a cargo de la Escuela de Terapia Ocupacional de la Universidad de Chile y la Unidad de Readaptación Social de Gendarmería de Chile representada por los Psicólogos Gonzalo Guzmán y Alejandra Acevedo, esto permitió definir los requerimientos técnicos y las necesidades concretas para la ejecución de la propuesta.

El módulo se implementó a través de dos fases:

- $\quad$ Fase de Diseño

- Fase de Implementación

En la Fase de Diseño la construcción del módulo implicó en primer lugar una revisión del estado del arte de la ciencia de la ocupación en relación a elementos conceptuales aplicables a contextos penitenciaros, además de recoger experiencias de intervención ocupacional sistematizada.

Paralelamente se realizaron entrevistas grupales con profesionales de Gendarmería de Chile con experiencia en talleres de intervención con personas privada de libertad. Los ejes principales de conversación se referían al contexto de intervención y a la exploración de la ocupación de las PPL. Las entrevistas fueron analizadas y los juicios de los expertos se incluyeron en el diseño del modelo y también en el manual de aplicación.

El trabajo de diseño del módulo implicó además la revisión de conceptos y su adaptación al contexto de intervención ${ }^{6}$. Este proceso se constituye en una herramienta fundamental para cohesionar la teoría con la práctica, en este sentido algunos de los conceptos más relevantes se expresan a continuación:

En relación a la Rutina Ocupacional se propone a los expertos la siguiente definición: "Conjunto de actividades desarrolladas en un día que se repiten continuamente o tienen un patrón común en un tiempo determinado, por ejemplo en una semana. Incluye actividades de autocuidado, tiempo libre, ocio, productividad y descanso". En relación a este tema se destaca la necesidad de estructurarlas y graduarlas de acuerdo a la situación carcelaria, se manifiesta que donde hay estructura y sentido las intervenciones resultan, también surge la necesidad de hacer quiebres con respecto a las rutinas institucionalizadas, destacando que

\footnotetext{
6 Los conceptos principales a trabajar fueron: Integración Social, Ocupación, Actividades Significativas, Flujo - Participación en la Actividad, Rutina Ocupacional, Vida Saludable, Participación Social, Solidaridad, Red Familiar-Social
} 
el cambio requiere recursos e infraestructura y también trabajar en lo formativo. Finalmente en el concepto modificado se conserva la definición inicial agregando: permitiendo un equilibrio ocupacional entendido como:

"Integración un número optimo de roles apropiados en la vida de un individuo. Un equilibrio de estos otorga ritmo y organización y se da cuando no están en conflicto o en competencia en el tiempo y cuando hay roles adecuados a la estructura del uso del tiempo.

Lograr un equilibrio en los roles contribuye a un desempeño ocupacional exitoso que a su vez aporta a la satisfacción en la vida."

En relación al concepto de Ocupación se entregan los siguientes lineamientos: "Se entiende como el grupo de actividades y tareas de cada día, las cuales son determinadas y organizadas. El valor y el significado que adquieren vienen dado por el individuo y su cultura. También se puede entender como todas las cosas que la gente hace para ocuparse, Incluyendo cuidar de sí mismo, disfrutar de su tiempo libre y contribuir para la construcción social y económica de su comunidad". En la discusión grupal se establece que se trataría de un área no trabajada habitualmente que abre territorios nuevos de la rehabilitación. Del punto de vista penitenciario nos encontraríamos con situaciones de privación ocupacional. Además se insiste que actualmente se observa que las PPL tienen mucho tiempo sin intervención institucional y de encierro con grupo de pares, con pocas alternativas ocupacionales. La mayoría no dispone de programas laborales. Algunos hacen artesanías, en forma precaria. Tendrían mucho tiempo destinado a sociabilidad y diversión. Idealmente la institución se orienta a favorecer escolarización y el área laboral. Finalmente en el concepto modificado se establece que: "El módulo se basa en el concepto de ocupación, a través de dispositivos de exploración de distintos tipos de actividades y ocupaciones. Que experimenten satisfacción ocupacional y flujo en la actividad. La estrategia se basa en el auto descubrimiento y la motivación intrínseca y en trabajar con la ocupación aquí y ahora., Se incluiría el diagnóstico de posibilidades ocupacionales (fisicas, mentales, sensoriales)"

Identificando el foco de la propuesta también se conceptualizó en torno a la Intervención Socio Ocupacional presentando la siguiente propuesta conceptual: "Orientada según un currículum personalizado. Basada en el análisis crítico de la ocupación. Apoya el desarrollo de competencias, de sentirse hábiles. Genera la oportunidad de desarrollar nuevas identidades a través de experiencias innovadoras.". En relación a esta propuesta los expertos señalaron que actualmente no hay tiempo disponible de los profesionales para talleres más complejos ni la infraestructura necesaria. Lo más completo es lo laboral y es para muy pocos. La larga socialización en comportamientos disóciales requieren intervenciones intensivas y hay que tener una baja expectativa de logros. En el contexto de reestructuración para hacer talleres intensivos, existe la necesidad de hacer intervención más allá de las actuales 4 horas disponibles. Para ello habría que cambiar reglamentos y tener más gendarmes, además se refiere que es posible una dificultad o choque con el régimen de castigos y disciplina interna gendarmería, para esto hay que trabajar los prejuicios de los funcionarios. En relación a las PPL existe valoración por cosas útiles, por adquirir un oficio, pero otros instrumentalizan el sistema, podría existir riesgo de simulación de participación. Para lograr resultados se requiere ser constantes y flexibles. Contemplar contingencias propias del funcionamiento carcelario. Además tomar en cuenta 
que las PPL se cansan rápido y se desconcentran, separar a los PPL por criterios criminológicos (respectar división de sectores) y contar con infraestructura adecuada.

Bajo estos hallazgos se propone: "intervención universal priorizando a los que necesitan más apoyo. Se basa en un análisis crítico vivencial o experiencial más que ideológico. Incluir lo corporal y no sólo lo verbal, debería existir la posibilidad de intervenir tanto en tiempos de encierro o desencierro. En definitiva constituye una verdadera reingeniería de los participantes, incluidos los propios profesionales. Puede incluir PPL como monitores. Implica la transferencia de saberes prácticos para participar en actividades. Es un proceso de habilitación no de rehabilitación, desde la motivación intrínseca sujeto."

Ya que el propósito final de la propuesta es favorecer la inclusión social de las PPL se establece la relación del concepto de Integración Social como: Adquisición de competencias habilitantes para la participación social, en diversos grados: motricidad, percepción, desempeño, etc.En cierto sentido todos los talleres en que participan PPL apuntarían a esto. Se propone un trabajo específico desde las actividades cotidianas de los sujetos. Así la discusión se genera en torno a que existe mucha gente sin redes familiares ni espacio en estructuras sociales /laborales, actualmente hay mitos sobre la inserción laboral al egreso, idealizando el trabajo, también se idealiza la familia y el futuro de los hijos. Existe posible desconfianza en la capacidad de funcionar en un trabajo normado externamente, al respecto deberían plantearse metas mínimas de intervención, orientadas a reducir la exclusión y facilitar las competencias. Una de ellas puede ser el desarrollo de la conciencia de bienes Públicos y el compartir de valores sociales. . Finalmente se modifica el concepto original definiendo que: "La integración social se orienta a retomar en forma personalizada aspectos de la socialización los organismos o instituciones sociales no abordaron. Implica restituir elementos de integración que le faltan al sujeto. Incluye el desarrollo de habilidades de para la participación en actividades sociales."

Todo este proceso dio origen a las "Cinco Unidades de Trabajo" que componen el módulo. En primer lugar se desarrollan conceptos relacionados con la ocupación y la rutina ocupacional. Posteriormente se aborda las implicancias de la incorporación de estilos de vida saludable y las ocupaciones. En continuidad con lo anterior, se realiza una aproximación al trabajo en proyectos solidarios, su efecto en la calidad de vida y la integración desde la participación. Por último, se entregan antecedentes para contextualizar el trabajo en familia, desde una forma congruente con el enfoque basado en la ocupación.

La primera Unidad, basada en el concepto de "Rutina Ocupacional" ese orienta al objetivo de que los participantes logren organizar una rutina o estructura ocupacional diaria satisfactoria y diversa que apoye la futura reinserción social. El concepto se trabaja en relación a rutina, AVD, importancia y función de las actividades, así como cambio de rutinas.

La segunda unidad "Exploraciones Ocupacionales" tiene por objetivo que los participantes vivencien actividades con diversas características encontrando satisfacción e interés en algunas de ellas. Consiste en un proceso individual y grupal de participación y vivencia en actividades con características o componentes especialmente definidos como sensoriomotores, cognitivos y espirituales. 
La tercera unidad "Vida Saludable" se orienta a promover estilos de vida saludable en las personas privadas de libertad permitiendo mejorar su calidad de vida, incorporando alguna actividad que promueva el cuidado de la salud. Se trabajo sobre un conjunto de conductas que se relacionan con el comportamiento de las personas respecto de la exposición a factores nocivos que representan riesgo para la salud. Incluye la alimentación, actividad física, enfermedades crónicas no transmisibles, consumo de tabaco, alcohol y autocuidado.

La cuarta unidad "Proyectos Ocupacionales Solidarios" busca que las personas privadas de libertad puedan diseñar un proyecto en grupo para promover el bienestar de sus compañeros, familia cercana o personas con necesidades determinadas. Con esto se busca potenciar el involucramiento en actividades significativas de tipo solidaria o que fomenten un estado de salud y bienestar en la población. Estos proyectos pasan por las etapas de conformación de grupos, planteamiento de problema y búsqueda de soluciones y diseño escrito del proyecto.

Las última unidad "fortaleciendo la familia" busca que los familiares de las personas privadas de libertad a través de las ocupaciones fortalezcan y valoren el vinculo como agentes apoyadores activos del proceso de integración socio ocupacional. A través de un proceso formativo, se busca aportar en la significación del rol de acompañante de los familiares en el proceso de integración socio ocupacional de las personas privadas de libertad. Ésta Unidad se implementa en el período previo y como parte de la preparación para el egreso de las personas privadas de libertad. Sus participantes son familiares u otras personas significativas para la persona privada de libertad.

Una vez definidos las unidades centrales de la intervención, el equipo fue desarrollando la estructura de las sesiones trabajo y la ruta del proceso, incorporando el aporte teórico de la Ciencia de la Ocupación y las metodologías de intervención con enfoque participativo, centrado en la reflexión y la motivación intrínseca.

El modelo propone que la persona privada de libertad tenga la oportunidad de recibir apoyo de un profesional “ Tutor" quién implementará la Ruta de Desarrollo Ocupacional Individual, que es la intervención directa e individual con un participante en particular, se espera que todos puedan acceder a esta instancia en alguna etapa de las unidades, esto dependerá del equipo profesional de la institución que realizará un análisis diagnostico para definir a los candidatos que estén preparados para recibir este beneficio. El objetivo central de esta ruta es que se encuentren estrategias en conjunto para implementar la participación en ocupaciones significativas, organizar rutinas, crear proyectos durante su periodo de reclusión, esto permitirá fortalecer las habilidades y proyectos trabajados en las unidades.

Cada PPL ingresa a alguna de las 4 primeras unidades, de acuerdo a las posibilidades de cada centro, en la quinta unidad de la familia participa una persona significativa de apoyo y es realizada próxima al egreso de la persona del sistema carcelario. Ver Figura 1.

Posteriormente en la Fase de Implementación se realizó la capacitación a 27 profesionales de Gendarmería de Chile, finalizando con la aplicación de algunas sesiones seleccionadas a 80 PPL, la aplicación a estas personas fue piloteada por un equipo externo que evaluó la funcionalidad, las dificultades que presentaba para los profesionales y la recepción por 
parte de las PPL, todo este proceso permitió testear el funcionamiento del módulo y realizar los ajustes necesarios.

Finalmente se procedió a la impresión final y entrega del documento.

Figura 1

PROCESO DE DESARROLLO DE LAS UNIDADES

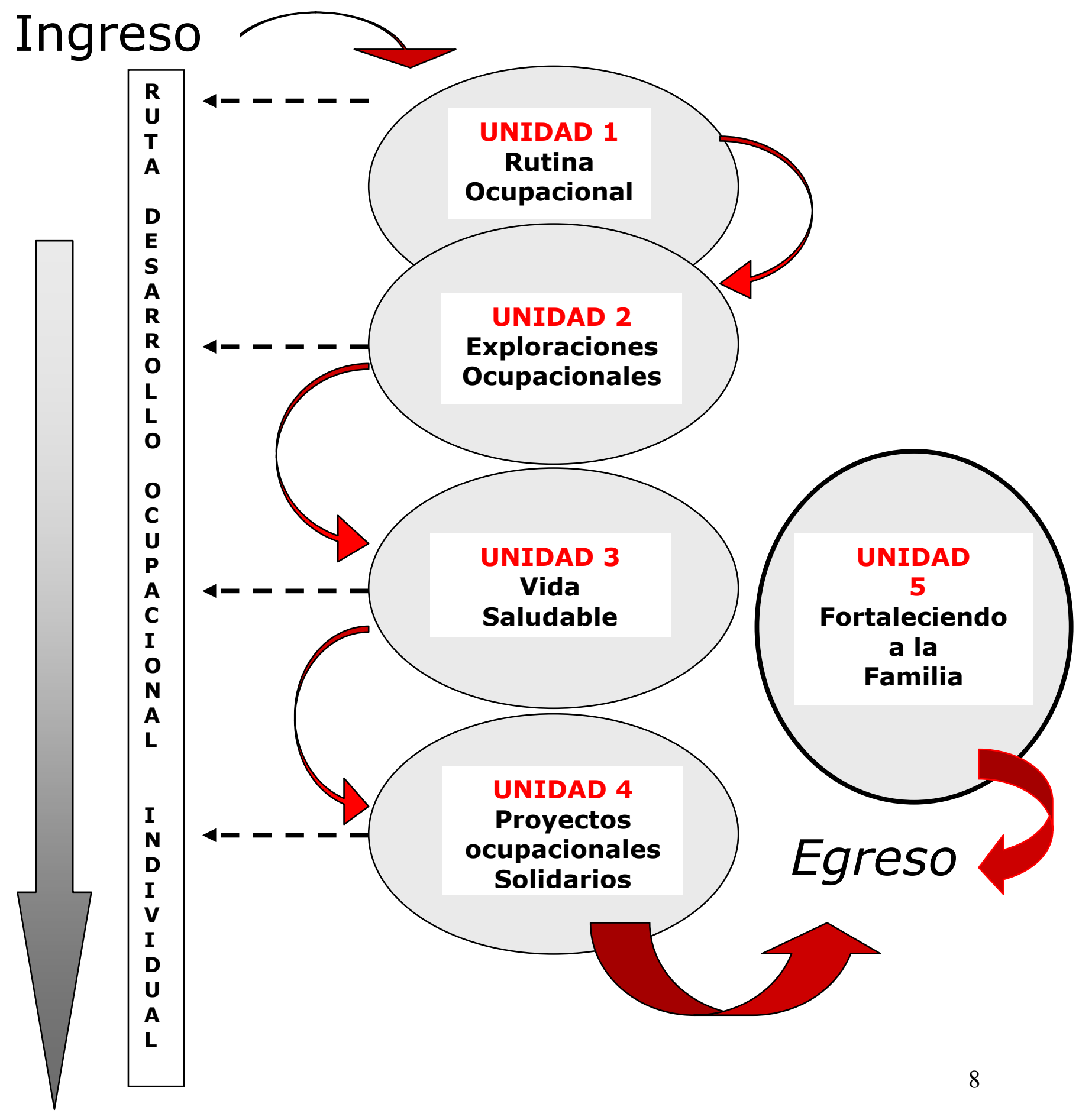




\section{DESCRIPCIÓN DEL MÓDULO OCUPARSE "Ocupación en personas privadas de libertad"}

El módulo apunta a la adquisición de habilidades socio ocupacionales como componente esencial para la posterior integración social de las personas privadas de libertad. Estas habilidades son adquiridas en un proceso continuo y secuenciado apoyado por los profesionales y funcionarios del sistema de Gendarmería y están sustentadas en un programa de reinserción integral del que constituyen una parte. En este escenario el Módulo de Integración Socio Ocupacional OCUPARSE es desarrollado fundamentalmente a través la participación y vivencia activa de las personas en las sesiones grupales que constituyen las unidades de trabajo del módulo.

En la aplicación del módulo pueden participar profesionales como: Terapeuta Ocupacional, Asistente Social o Psicólogo, que cuentan con competencias para el manejo de grupo, en caso de requerir aportes o manejo específicos de cada línea profesional deben ser derivados a los especialistas correspondientes respetando su experticia profesional:

Terapeuta Ocupacional: alteraciones del desempeño ocupacional, rutina y equilibrio ocupacional, inserción laboral y evaluaciones de puestos de trabajo, apoyo a la integración escolar, atención de Personas con Discapacidad, desarrollo de habilidades neuromotrices, de procesamiento cognitivo y relación social e interacción entre otras.

Asistente Social: trabajo con la familia, trabajo en redes, contacto con la comunidad, evaluación de beneficios sociales, etc.

Psicólogo: alteraciones de la conducta, problemas de situación de vida, desarrollo de habilidades psicológicas, evaluaciones específicas y otras.

Los profesionales deben pasar inicialmente por una etapa de capacitación que les permita comprender la organización interna del texto y realizar un estudio de sus bases conceptuales y fundamentos. La implementación y el aprendizaje del proceso de intervención se ve favorecido por las características centrales de este, que son: su facilidad de aplicación ya que cuenta con la descripción detallada del procedimiento y los materiales didácticos, su coherencia interna respaldada en el análisis de los fundamentos conceptuales y la aplicación de etapas previas de validación y la flexibilidad que permite una implementación dinámica sin poner en riesgo su congruencia.

A la capacitación se pueden sumar los monitores de cada institución (monitores laborales, monitores de talleres artesanales, etc.) aunque ellos no participen directamente en la intervención es importante que conozcan la estructura del módulo y de las sesiones, para que puedan apoyar en forma activa el proceso en coherencia con los objetivos planteados.

El Módulo está organizado en tres secciones:

1.- FUNDAMENTOS CONCEPTUALES que orientan la intervención, en esta sección los conceptos teóricos están divididos de acuerdo a las unidades de trabajo y cuenta con sus respectivas referencias bibliográficas. 
2.- UNIDADES DE TRABAJO: corresponde al plan de intervención estructurado en 5 unidades de trabajo ${ }^{7}$, en cuatro de ellas participan las personas privadas de libertad y en la quinta un familiar u otro significativo.

Cada unidad consta de 10 sesiones a excepción de la unidad 5 que cuenta con 8 sesiones, cada sesión de trabajo tiene una duración aproximada de 60 minutos, o de 45 en el caso de las sesiones con la familia u otra persona significativa. En este último caso la actividad dura menos tiempo para dejar tiempo para el contacto libre durante la visita.

El modulo está diseñado para que el participante pueda ingresar a cualquiera de las unidades pero siempre a la primera sesión, esto permite incluir en las actividades a personas en forma desfasada. Con esto se busca flexibilizar la participación de las PPL, del mismo modo se consideró que la cantidad de sesiones por semana dependerá de la realidad del centro, pudiendo ser aplicadas por ejemplo 2 o 3 veces por semana una unidad o paralelamente 2 unidades.

La unidad que incluye a la familia esta se implementa al aproximarse el egreso de la persona para apoyar la salida la persona privada de libertad. Esta unidad se realiza utilizando el tiempo de espera, previo a las visitas establecidas por el centro de reclusión.

Cada unidad de trabajo incluye los siguientes componentes:

- Definición: Especifica los conceptos involucrados en la unidad, hace referencia a la comprensión del tema desde la perspectiva del módulo.

- Objetivo General: Incluye las metas o aspiraciones finales de la unidad.

- Actividades: Cuadro resumen que indica los nombres de cada una de las sesiones.

- Sesiones de Trabajo: Cada unidad cuenta con un número de sesiones que son actividades de intervención específicas.

3.- METODOLOGÍA DE EVALUACIÓN: considera la evaluación que permite medir los impactos y resultados, esta evaluación debe ser constante y contener registros claros de las actividades realizadas en forma continua. La metodología para evaluar los resultados implica la realización de un Cuestionario de Percepción Ocupacional aplicado al inicio y al final, una Matriz de Indicadores de Sesiones que permitirá ver la secuencia de aplicación y la construcción de un Portafolio Reflexivo de cada participante. Las definiciones de cada elemento son:

\footnotetext{
${ }^{7}$ Unidad Rutina Ocupacional, Unidad Exploraciones Ocupacionales, Unida Vida Saludable, Unidad Proyectos Ocupacionales Solidarios y Unidad Fortaleciendo a la Familia.
} 
Matriz de Indicadores de Sesiones: es una Planilla de registro por sesión, contiene logros desde la perspectiva cuantitativa, específicamente el porcentaje por cada indicador, por participante y total. Además incluye un espacio reservado para las observaciones que constituyen un aporte a la evaluación como antecedentes cualitativos u observacionales.

Portafolio del participante: es una estrategia de aprendizaje y concientización de las vivencias, puede ser una carpeta o maletín en donde cada participante incluye los registros o evidencias de sus sesiones en forma organizada.

Cuestionario de Percepción Ocupacional: es un Cuestionario de evaluación aplicado en la primera unidad (recuerde que esta es variable) y la última unidad, que arroja información acerca de las condiciones de entrada y salida del participante.

\section{Conclusiones}

El Módulo de Ocupación Socio Ocupacional para Personas Privadas de Libertad, constituye una experiencia inédita en nuestro país. Contribuye al mejoramiento de las condiciones de vida de los sujetos reclusos y al mismo tiempo que favorece procesos de integración social.

Diseñamos este módulo partiendo de la base que la intervención basada en la actividad y la ocupación como dimensión teórica, pueden aportar de manera significativa al bienestar y a los procesos de integración social de las personas privadas de libertad. Por otro lado este módulo instala la perspectiva de la ocupación en un ámbito donde el trabajo de los terapeutas ocupacionales es aún incipiente y con instrumentos como éste potencia sus posibilidades de acción.

Por otro lado, esperamos en el futuro poder realizar una evaluación de los resultados del módulo y aportar a su mejoramiento.

Por último, queremos señalar que este trabajo demuestra que es posible contribuir desde la academia en aspectos estratégicos del desarrollo de nuestro país desde una perspectiva pública. Lo cual es parte de la misión de nuestra Universidad y nuestra Escuela. Queremos agradecer Gendarmería de Chile, por habernos dado la oportunidad de llevar adelante este trabajo y haber generado las condiciones para que sea aplicado en los recintos penitenciaros. 


\section{BIBLIOGRAFÌA}

- Asociación Americana de Terapia Ocupacional (2002). Marco de Trabajo para la Práctica de la Terapia Ocupacional: dominio y proceso. Revista Americana de Terapia Ocupacional (AJOT), 56, 609-639.

- Crepeau E., Cohn E., Schell B. 2003. Willard \& Spackman's Terapia Ocupacional. $10^{\mathrm{a}}$ Ed. Lippincott, Williams \& Wilkins. Philadelphia.

- Gómez S, Rueda, L. \& Muñoz, C. (2002). Patrón de Idiosincrasia de los alumnos que ingresan a Terapia Ocupacional. En: Bases para el estudio de la Ciencia de la Ocupación (pp 87-94). Santiago: Facultad de Medicina. Universidad de Chile.

- Towsend, E. \& Wilcock, A. (2002) Occupational justice. En Christiansen, Ch. \& Townsend, E. Introduction to occupation (pp243-275) Thorofare, NJ: Prentice Hall.

- Westorp, P. (2003) Balance exploring as a concept in Occupational Science. Journal of Occupational Science, 10 (2). 